\section{Stabilization of Markovian Systems via Probability Rate Synthesis and Output Feedback}

\author{
Jun-E Feng, James Lam, and Zhan Shu
}

\begin{abstract}
This technical note is concerned with the stabilization problem of Markovian jump linear systems via designing switching probability rate matrices and static output-feedback gains. A novel necessary and sufficient condition is established to characterize the switching probability rate matrices that guarantee the mean square stability of Markovian jump linear systems. Based on this, a necessary and sufficient condition is provided for the existence of desired controller gains and probability rate matrices. Extensions to the polytopic uncertain case are also provided. All the conditions are formulated in terms of linear matrix inequalities with some equality constraints, which can be solved by two modified cone complementarity linearization algorithms. Examples are given to show the effectiveness of the proposed method.
\end{abstract}

Index Terms-Linear matrix inequality (LMI), Markovian process, output feedback, stabilization, switched system.

\section{INTRODUCTION}

Markovian jump linear systems, which were first introduced in [1], are modeled by a set of linear plants with transitions among them according to a Markov chain taking values in a finite set. This kind of model can capture the abrupt changes that appear in the system structure or its parameters, and has found widespread applications in various practical problems such as target tracking, manufacturing processes, and fault-tolerant control systems [2].

Over the past decades, a variety of results about Markovian jump linear systems have been obtained. Stability and stabilization have been studied thoroughly in [2]-[7], and references therein. $H_{\infty}$ control for Markovian jump systems has been reported in different cases, for instance, continuous-time [8], discrete-time [9], bilinear system [10], time-delay [11], [12], descriptor system [13], and two-dimensional system [14]. The quadratic optimal control problem concerning these systems has been discussed in [15] and [16]. The filtering problem for the continuous-time case has been investigated in [12], [17] and [18]. For a class of discrete-time Markov jump linear systems with partially known transition probabilities, stability analysis and stabilization synthesis problems have been considered in [19] and [20], respectively, for systems without delay and for those with time-varying delays.

It should be pointed out that almost all the results mentioned above were derived for given switching probability rate matrices except [19] and [20]. In some practical cases, however, designers may have freedom to choose appropriate switching probability rate matrices or general switching rules. Some motivations for this and more general

Manuscript received March 30, 2009; revised June 29, 2009. First published February 08, 2010; current version published March 10, 2010. This work was supported in part by RGC Grant HKU 7031/06P, the National Natural Science Foundation of China under Grant 60974137, and by the Science Foundation of Ireland 07/PI/I1838. Recommended by Associate Editor P. Colaneri.

J.-E. Feng is with the School of Mathematics, Shandong University, Jinan 250100, China (e-mail: fengjune@sdu.edu.cn).

J. Lam is with the Department of Mechanical Engineering, The University of Hong Kong, Hong Kong, China (e-mail: james.lam@hku.hk).

Z. Shu was with the Department of Mechanical Engineering, The University of Hong Kong, Hong Kong, China. He is now with the Hamilton Institute, National University of Ireland, Maynooth, Ireland (e-mail: shuzhan@graduate.hku.hk).

Color versions of one or more of the figures in this technical note are available online at http://ieeexplore.ieee.org.

Digital Object Identifier 10.1109/TAC.2010.2040499 hybrid output-feedback control have been presented in [21]. In [21], stabilizing switching signals have been constructed when none of the individual subsystems is asymptotically stable. Closed-loop and open-loop switching control strategies have been investigated in [22] and [23]. State-feedback and output-feedback switching design have been considered in [27], [28]. Although these results are nice, they are mainly concerned with deterministic switching, and little effort has been made towards stochastic switching. The selection or determination of stochastic switching law, to a large extent, remains an open problem.

In this technical note, we shall first present a novel characterization for switching probability rate matrices that render mean square stability, and design such a matrix. This complements and differs from the work in [19] where the transition matrices in discrete-time are partially known or the work in [7] where the probability rate matrices have element-wise uncertainties. Based on this, a necessary and sufficient for the existence of desired static output-feedback (SOF) gains and probability rate matrices is provided. The corresponding results are extended to the polytopic uncertain case. All the results are formulated in terms of linear matrix inequalities (LMIs) with some matrix equality constraints, which can be solved by two modified cone complementarity linearization (CCL) algorithms inspired by [24].

Notation: Throughout this technical note, $\mathbb{R}^{n}$ represents the $n$-dimensional Euclidean space. The notation $\|\cdot\|$ refers to Euclidean norm for vector. $\mathbf{E}$ is the mathematical expectation operator. We use superscript $T$ and symbol $\operatorname{tr}(\cdot)$ for the transpose and trace of a matrix, respectively. For real symmetric matrices $P$ and $Q$, the notation $P>Q$ (respectively, $P \geq Q$ ) means $P-Q$ is positive definite (respectively, positive semidefinite). $I$ is the identity matrix with compatible dimensions. Matrices, if their dimensions are not explicitly stated, are assumed to have compatible dimensions.

\section{PReliminaries AND PRoblem Statement}

Consider the following linear continuous-time system with Markovian jump parameters:

$$
\left\{\begin{array}{l}
\dot{x}(t)=A(r(t)) x(t)+B(r(t)) u(t), \\
y(t)=C(r(t)) x(t), \\
x(0)=x_{0} \in \mathbb{R}^{n}, \quad r(0)=r_{0}
\end{array}\right.
$$

where $x(t) \in \mathbb{R}^{n}, u(t) \in \mathbb{R}^{m}$, and $y(t) \in \mathbb{R}^{p}$ are the system state, the control input, and the measured output, respectively. $\{r(t), t \geq 0\}$ is a continuous-time Markov process taking values in the finite discrete set $S=\{1,2, \ldots, N\}$. For $r(t)=i, A(r(t)), B(r(t)), C(r(t))$ are given constant matrices, denoted by $A_{i}, B_{i}$ and $C_{i}$ for simplicity. The mode transition probabilities are described as follows:

$$
\operatorname{Pr}(r(t+\Delta)=j \mid r(t)=i)= \begin{cases}\lambda_{i j} \Delta+o(\Delta), & j \neq i, \\ 1+\lambda_{i j} \Delta+o(\Delta), & j=i\end{cases}
$$

where $\Delta>0, \lambda_{i j} \geq 0, \forall j \neq i$, and $\lambda_{i i}=-\sum_{j \neq i} \lambda_{i j}<0$, and $o(\Delta) / \Delta \rightarrow 0$ as $\Delta$ approaches to zero. Denote $\Pi=\left(\lambda_{i j}\right)_{N \times N}$ (a square matrix is said to be Metzler if its off-diagonal entries are nonnegative).

Throughout this technical note, we will use the following definition on mean square stability for system (1) (see [4]).

Definition 1: The free system (1) (that is, $u(t) \equiv 0$ ) is said to be mean square stable if

$$
\mathbf{E}\left[\|x(t)\|^{2}\right] \rightarrow 0, \quad \text { as } \quad t \rightarrow \infty
$$

for any initial condition $x_{0} \in \mathbb{R}^{n}$ and initial distribution $r_{0}$. 
In this technical note, we shall first establish a necessary and sufficient condition on the choice of the probability rate matrix for mean square stability of free system (1). Then we will design a mode-dependent SOF (MDSOF) controller

$$
u(t)=K(r(t)) y(t)
$$

and a probability rate matrix such that the resulting closed-loop system is mean square stable.

To this end, we need to introduce the following stability lemma which will be used in the sequel.

Lemma 1: [25]: For a given probability rate matrix $\Pi$, free system (1) is mean square stable if and only if there exists a set of positive definite matrices $P_{1}, P_{2}, \ldots, P_{N}$ satisfying the following inequalities:

$$
A_{i}^{T} P_{i}+P_{i} A_{i}+\sum_{j=1}^{N} \lambda_{i j} P_{j}<0, \quad i=1,2, \ldots, N .
$$

For a given probability rate matrix $\Pi$, the inequalities in (5) are linear on the positive definite matrices $P_{i}$. When the probability rate matrix $\Pi$ is not known, the stability characterization (5) turns out to be bilinear due to the product terms of the positive definite matrices $P_{i}$ and the elements in $\Pi$. If inequalities (5) are feasible for a given probability rate matrix $\Pi$, it can be shown that there exists a stable convex combination of the given matrices $A_{i}$. However, checking the existence of a stable combination is an NP-hard problem. In this technical note we manage to rewrite the problem as LMIs with some equality constraints and present an algorithm to solve it partially.

\section{Stabilizing Probability Rate Matrices}

In this section, we consider the choice of a probability rate matrix, which ensures the stability of free system (1). Firstly, we need to find a means to separate matrix variables $P_{i}$ and elements of the probability rate matrix $\Pi$. Motivated by [26], we handle this separation problem in the following theorem, which transforms the nonlinear problem into linear one with some matrix equality constraints.

Theorem 1: There exists a probability rate matrix such that free system (1) is mean square stable if and only if there exist a set of positive definite matrices $X_{1}, X_{2}, \ldots, X_{N}, \bar{X}_{1}, \bar{X}_{2}, \ldots, \bar{X}_{N}$, a scalar $\varepsilon>0$, and a Metzler matrix $\bar{\Pi}=\left(\bar{\lambda}_{i j}\right)_{N \times N}$, satisfying

$$
\left[\begin{array}{ccc}
-X_{i}-\sum_{j=1}^{N} X_{j} & \Upsilon_{i} & I+\varepsilon A_{i}^{T} \\
* & -\Phi & 0 \\
* & * & -\bar{X}_{i}
\end{array}\right]<0
$$

and

$$
X_{i} \bar{X}_{i}=I
$$

where $\Upsilon_{i}=\left[\begin{array}{llll}\left(1+\bar{\lambda}_{i 1}\right) I & \left(1+\bar{\lambda}_{i 2}\right) I & \ldots & \left(1+\bar{\lambda}_{i N}\right) I\end{array}\right]$, $\Phi=\operatorname{diag}\left\{\bar{X}_{1}, \bar{X}_{2}, \ldots, \bar{X}_{N}\right\}$ and $i=1,2, \ldots, N$. In this case, a stabilizing probability rate matrix is given by $\Pi=2 / \varepsilon \bar{\Pi}$.
Proof: There exists a probability rate matrix $\Pi$ such that free system (1) is mean square stable if and only if there exists a set of positive definite matrices $P_{1}, P_{2}, \ldots, P_{N}$ satisfying the inequalities in (5). On the other hand, the inequalities in (5) hold if and only if there exists a sufficient small scalar $\varepsilon>0$ such that the following inequalities hold:

$$
\begin{aligned}
A_{i}^{T} P_{i}+P_{i} A_{i}+\sum_{j=1}^{N} \frac{\lambda_{i j}}{2} P_{j} & +\sum_{j=1}^{N} \frac{\lambda_{i j}}{2} P_{j} \\
& +\varepsilon \sum_{j=1}^{N} \frac{\lambda_{i j}}{2} P_{j} \frac{\lambda_{i j}}{2}+\varepsilon A_{i}^{T} P_{i} A_{i}<0 .
\end{aligned}
$$

By Schur complement equivalence, it is easy to see that the inequalities in (8) hold if and only if the following inequalities hold:

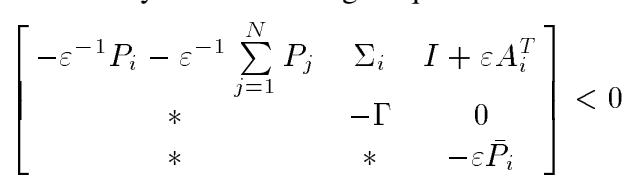

where $\bar{P}_{i}=P_{i}^{-1}, \Gamma=\operatorname{diag}\left\{\varepsilon \bar{P}_{1}, \varepsilon \bar{P}_{2}, \ldots, \varepsilon \bar{P}_{N}\right\}$ and $\Sigma_{i}=[(1+$ $\left.\left.\varepsilon / 2 \lambda_{i 1}\right) I\left(1+\varepsilon / 2 \lambda_{i 2}\right) I \ldots\left(1+\varepsilon / 2 \lambda_{i N}\right) I\right]$. Taking $X_{i}=\varepsilon^{-1} P_{i}$, $\bar{X}_{i}=\varepsilon \bar{P}_{i}$ and $\bar{\lambda}_{i j}=\varepsilon / 2 \lambda_{i j}$ in the above inequalities, it is clear that the inequalities in (9) hold if and only if the inequalities in (6) and the matrix equalities in (7) hold. Thus the proof is completed.

The result in Theorem 1 also holds when the free system (1) has polytopic uncertainties, that is

$$
A_{i}=\sum_{m=1}^{s} \alpha_{m} A_{i m}, \quad \sum_{m=1}^{s} \alpha_{m}=1, \quad \alpha_{m} \geq 0
$$

which is described in the following corollary.

Corollary 1: For free system (1) with polytopic uncertainties (10), there exists a probability rate matrix such that the system is mean square stable if and only if a set of positive definite matrices $X_{1}, X_{2}, \ldots, X_{N}, \bar{X}_{1}, \bar{X}_{2}, \ldots, \bar{X}_{N}$, a scalar $\varepsilon>0$, and a Metzler matrix $\bar{\Pi}=\left(\bar{\lambda}_{i j}\right)_{N \times N}$, satisfying

$$
\Xi_{i m}=\left[\begin{array}{ccc}
-X_{i}-\sum_{j=1}^{N} X_{j} & \Upsilon_{i} & I+\varepsilon A_{i m}^{T} \\
* & -\Phi & 0 \\
* & * & -\bar{X}_{i}
\end{array}\right]<0
$$

and the equalities in (7), where $\Upsilon_{i}$ and $\Phi$ are defined in Theorem 1, where $i=1,2, \ldots, N ; m=1,2, \ldots, s$. In this case, a stabilizing probability rate matrix is given by $\Pi=2 / \varepsilon \bar{\Pi}$.

Proof: The necessity is obvious, so here we only give the proof of sufficiency. Noting that $\alpha_{m} \geq 0$ and $\sum_{m=1}^{s} \alpha_{m}=1$, from (11) we have $\sum_{m=1}^{s} \alpha_{m} \Xi_{i m}<0$, that is (12), shown at the bottom of the page, where $i=1,2, \ldots, N$. With $\sum_{m=1}^{s} \alpha_{m} A_{i m}=A_{i}$ and $\sum_{m=1}^{s} \alpha_{m}=1$, (6) is obtained from (12). Then using Theorem 1, we derive the stability of uncertain free system (1). Thus the proof is completed.

$$
\left[\begin{array}{ccc}
-\sum_{m=1}^{s} \alpha_{m}\left(X_{i}+\sum_{j=1}^{N} X_{j}\right) & \sum_{m=1}^{s} \alpha_{m} \Upsilon_{i} & \sum_{m=1}^{s} \alpha_{m}\left(I+\varepsilon A_{i m}^{T}\right) \\
* & -\sum_{m=1}^{s} \alpha_{m} \Phi & 0 \\
* & * & -\sum_{m=1}^{s} \alpha_{m} \bar{X}_{i}
\end{array}\right]<0
$$


It should be pointed out that the conditions in Theorem 1 and Corollary 1 are presented by a set of LMIs with some matrix equality constraints, which cannot be solved directly with standard software due to their non-convex nature. However, we can tackle them via employing the CCL algorithm.

The basic idea of CCL algorithm is that if

$$
\left[\begin{array}{cc}
X_{i} & I \\
I & \bar{X}_{i}
\end{array}\right] \geq 0
$$

is solvable in $n \times n$ matrices $X_{i}>0$ and $\bar{X}_{i}>0$, then $\operatorname{tr}\left(X_{i} \bar{X}_{i}\right) \geq n$, while $\operatorname{tr}\left(X_{i} \bar{X}_{i}\right)=n$ if and only if $X_{i} \bar{X}_{i}=I$. Therefore, we summarize a schematic algorithm to solve the original non-convex feasibility problem formulated in Theorem 1.

Stabilizing Probability Rate Matrix (SPRM) Algorithm:

1) For the inequalities in (6), find a set of feasible positive definite matrices $\left(X_{1}^{0}, \bar{X}_{1}^{0}, X_{2}^{0}, \bar{X}_{2}^{0}, \ldots, X_{N}^{0}, \bar{X}_{N}^{0}\right)$. If there are none, EXIT. Set $h=0$.

2) For matrices $X_{1}^{h}, \bar{X}_{1}^{h}, X_{2}^{h}, \bar{X}_{2}^{h}, \ldots, X_{N}^{h}, \bar{X}_{N}^{h}$, solve the LMI problem: $\min \left\{\sum_{i=1}^{N} \operatorname{tr}\left(X_{i} \bar{X}_{i}^{h}+X_{i}^{h} \bar{X}_{i}\right)\right\}$ subject to (6) and (13), obtain $X_{1}, \bar{X}_{1}, X_{2}, \bar{X}_{2}, \ldots, X_{N}, \bar{X}_{N}, \bar{\Pi}$ and $\varepsilon$.

3) Taking $P_{i}=\varepsilon X_{i}$, and $\Pi=2 / \varepsilon \bar{\Pi}$, substitute the obtained variables $\left(P_{1}, P_{2}, \ldots, P_{N}, \Pi, \varepsilon\right)$ into (8) or (5). If condition (8) or (5) is satisfied, then the stabilizing probability rate matrix is $\Pi=$ $2 / \varepsilon \bar{\Pi}$. EXIT.

4) If $h>N_{0}$ where $N_{0}$ is the maximum number of iterations allowed, EXIT.

5) Set $h=h+1$, let $\left(X_{1}^{h}, \bar{X}_{1}^{h}, X_{2}^{h}, \bar{X}_{2}^{h}, \ldots, X_{N}^{h}, \bar{X}_{N}^{h}\right)=$ $\left(X_{1}, \bar{X}_{1}, X_{2}, \bar{X}_{2}, \ldots, X_{N}, \bar{X}_{N}\right)$, and go to Step 2).

Remark 1: The convergence of the algorithm can be shown by following a similar line as used in [24]. It is noted that, similar to CCL, the algorithm generally does not guarantee finding a globally optimal solution. For the computational complexity of the algorithm, on one hand, the optimization of each iteration is a convex optimization problem, of which the computational complexity with the interior-point method is polynomial time. On the other hand, the number of total iterative steps is bounded. Therefore, the computational complexity of the algorithm is also polynomial time.

Example 1: When the system matrices in free system (1) are

$$
\begin{aligned}
A_{1} & =\left[\begin{array}{cc}
0 & -1 \\
-2 & 0
\end{array}\right], \quad A_{2}=\left[\begin{array}{cc}
-1 & -1 \\
3 & 0
\end{array}\right], \\
\Pi & =\left[\begin{array}{cc}
-2 & 2 \\
5 & -5
\end{array}\right]
\end{aligned}
$$

we can check that inequalities in (5) are not solvable for variables $P_{1}$ and $P_{2}$ using Lemma 1 . Therefore the system above is not mean square stable for the given probability rate matrix $\Pi$. However, using algorithm SPRM, and solving inequalities in (6) and equalities in (7), we derive a stabilizing probability rate matrix

$$
\Pi=\left[\begin{array}{cc}
-19.1582 & 19.1582 \\
11.3426 & -11.3426
\end{array}\right] \text {. }
$$

\section{MDSOF DESIGN}

Based on the discussion in Section III, in this section we simultaneously design an MDSOF stabilizing controller and a probability rate matrix for system (1). When a control law (4) is applied to system (1), the closed-loop system becomes

$$
\dot{x}(t)=(A(r(t))+B(r(t)) K(r(t)) C(r(t))) x(t) .
$$

Now using Lemma 1, we provide a necessary and sufficient condition for the existence of MDSOF stabilizing controllers and probability rate matrices.

Theorem 2: There exist an MDSOF controller and a probability rate matrix such that the closed-loop system of (1) is mean square stable if and only if there exist a set of positive definite matrices $X_{1}, X_{2}, \ldots, X_{N}, \bar{X}_{1}, \bar{X}_{2}, \ldots, \bar{X}_{N}$, matrices $\bar{K}_{1}, \bar{K}_{2}, \ldots, \bar{K}_{N}$, a scalar $\varepsilon>0$, and a Metzler matrix $\bar{\Pi}=\left(\bar{\lambda}_{i j}\right)_{N \times N}$, satisfying

$$
\left[\begin{array}{cccc}
-2 X_{i}-\sum_{j=1}^{N} X_{j} & \Upsilon_{i} & I+C_{i}^{T} \bar{K}_{i}^{T} B_{i}^{T} & I+\varepsilon A_{i}^{T} \\
* & -\Phi & 0 & 0 \\
* & * & -\bar{X}_{i} & 0 \\
* & * & * & -\bar{X}_{i}
\end{array}\right]<0
$$

and the matrix equalities in (7), where $\Upsilon_{i}$ and $\Phi$ are defined in Theorem 1 , where $i=1,2, \ldots, N$. Then an MDSOF stabilizing controller (4) and the corresponding probability rate matrix are obtained, where $K_{i}=\varepsilon^{-1} \bar{K}_{i}$ and $\Pi=2 / \varepsilon \bar{\Pi}$.

Proof: From Lemma 1, there exist an SOF controller (4) and a probability rate matrix $\Pi$ such that closed-loop system (14) is mean square stable if and only if there exists a set of positive definite matrices $P_{1}, P_{2}, \ldots, P_{N}$ satisfying the following inequalities:

$$
\left(A_{i}+B_{i} K_{i} C_{i}\right)^{T} P_{i}+P_{i}\left(A_{i}+B_{i} K_{i} C_{i}\right)+\sum_{j=1}^{N} \lambda_{i j} P_{j}<0 .
$$

The above inequalities in (16) hold if and only if there exists a small scalar $\varepsilon>0$ such that the following inequalities hold:

$$
\begin{aligned}
& \left(A_{i}+B_{i} K_{i} C_{i}\right)^{T} P_{i}+P_{i}\left(A_{i}+B_{i} K_{i} C_{i}\right)+\sum_{j=1}^{N} \lambda_{i j} P_{j} \\
& +\varepsilon\left(B_{i} K_{i} C_{i}\right)^{T} P_{i}\left(B_{i} K_{i} C_{i}\right)+\varepsilon A_{i}^{T} P_{i} A_{i} \\
& +\varepsilon \sum_{j=1}^{N} \frac{\lambda_{i j}}{2} P_{j} \frac{\lambda_{i j}}{2}<0 .
\end{aligned}
$$

The remaining part of the proof is similar to Theorem 1, hence they are omitted here.

Similar to Theorem 1, Theorem 2 can be extended to the polytopic uncertain case. Assume that system (1) has polytopic uncertainties, that is

$$
\begin{aligned}
\left\{A_{i}, B_{i}\right\} & =\sum_{m=1}^{s} \alpha_{m}\left\{A_{i m}, B_{i m}\right\}, \quad \sum_{m=1}^{s} \alpha_{m}=1, \quad \alpha_{m} \geq 0 \\
C_{i} & =\sum_{r=1}^{l} \beta_{r} C_{i r}, \quad \sum_{r=1}^{l} \beta_{r}=1, \quad \beta_{r} \geq 0 .
\end{aligned}
$$

We have the following result for uncertain system (1).

Corollary 2: For system (1) with polytopic uncertainties (18) and (19), there exist an MDSOF controller and a probability rate matrix such that the closed-loop of system (1) is mean square stable if and only if there exist a set of positive definite matrices $X_{1}, X_{2}, \ldots, X_{N}, \bar{X}_{1}, \bar{X}_{2}, \ldots, \bar{X}_{N}$, matrices $\bar{K}_{1}, \bar{K}_{2}, \ldots, \bar{K}_{N}$, a scalar $\varepsilon>0$, and a Metzler matrix $\bar{\Pi}=\left(\bar{\lambda}_{i j}\right)_{N \times N}$, satisfying

$$
\begin{gathered}
\Phi_{i m r}= \\
{\left[\begin{array}{cccc}
-2 X_{i}-\sum_{j=1}^{N} X_{j} & \Upsilon_{i} & I+C_{i r}^{T} \bar{K}_{i}^{T} B_{i m}^{T} & I+\varepsilon A_{i m}^{T} \\
* & -\Phi & 0 & 0 \\
* & * & -\bar{X}_{i} & 0 \\
* & * & * & -\bar{X}_{i}
\end{array}\right]<0}
\end{gathered}
$$

and the matrix equalities in (7), where $\Sigma_{i}$ and $\Gamma$ are defined in Theorem 1 , and $i=1,2, \ldots, N, m=1,2, \ldots, s, r=1,2, \ldots, l$. In this case, 
an MDSOF stabilizing controller (4) and the corresponding probability rate matrix are obtained, where $K_{i}=\varepsilon^{-1} \bar{K}_{i}$ and $\Pi=2 / \varepsilon \bar{\Pi}$.

Proof: Similar to Corollary 1, here we only give the proof of sufficiency. Noting that $\alpha_{m} \geq 0$ and $\sum_{m=1}^{s} \alpha_{m}=1$, from (20) we have $\sum_{m=1}^{s} \alpha_{m} \Phi_{i m r}<0$, that is (21), shown at the bottom of the page, where $i=1,2, \ldots, N, \quad r=1,2, \ldots, l$. With $\sum_{m=1}^{s} \alpha_{m} A_{i m}=$ $A_{i}, \sum_{m=1}^{s} \alpha_{m} B_{i m}=B_{i}$ and $\sum_{m=1}^{s} \alpha_{m}=1$, from (21) we have See equation (22) at the bottom of the page.

$\Psi_{i r}=\left[\begin{array}{cccc}-2 X_{i}-\sum_{j=1}^{N} X_{j} & \Upsilon_{i} & I+C_{i r}^{T} \bar{K}_{i}^{T} B_{i}^{T} & I+\varepsilon A_{i}^{T} \\ * & -\Phi & 0 & 0 \\ * & * & -\bar{X}_{i} & 0 \\ * & * & * & -\bar{X}_{i}\end{array}\right]<0$.

Using $\sum_{r=1}^{l} \beta_{r} C_{i r}=C_{i}$ and $\sum_{r=1}^{l} \beta_{r}=1$, the remaining part of the proof can be easily obtained and hence it is omitted here for brevity. $\square$

To solve the non-convex feasibility problem formulated in Theorem 2, an algorithm similar to that of Theorem 1 can be constructed for deriving the MDSOF stabilizing controller and the corresponding probability rate matrix simultaneously.

Simultaneous SOF and Probability Rate Matrix (SOFPMR) Algorithm:

1) For the inequalities in (15), find a set of feasible positive definite matrices $\left(X_{1}^{0}, \bar{X}_{1}^{0}, X_{2}^{0}, \bar{X}_{2}^{0}, \ldots, X_{N}^{0}, \bar{X}_{N}^{0}\right)$. If there are none, EXIT. Set $h=0$.

2) For matrices $X_{1}^{h}, \bar{X}_{1}^{h}, X_{2}^{h}, \bar{X}_{2}^{h}, \ldots, X_{N}^{h}, \bar{X}_{N}^{h}$, solve the LMI problem: $\min \left\{\sum_{i=1}^{N} \operatorname{tr}\left(X_{i} \bar{X}_{i}^{h}+X_{i}^{h} \bar{X}_{i}\right)\right\}$ subject to (15) and (13), obtain $X_{1}, \bar{X}_{1}, X_{2}, \bar{X}_{2}, \ldots, X_{N}, \bar{X}_{N}, \bar{\Pi}$, $\bar{K}_{1}, \bar{K}_{2}, \ldots, \bar{K}_{N}$ and $\varepsilon$.

3) Taking $P_{i}=\varepsilon X_{i}, K_{i}=\varepsilon^{-1} \bar{K}_{i}$ and $\Pi=2 / \varepsilon \bar{\Pi}$, substitute the obtained variables $\left(P_{1}, P_{2}, \ldots, P_{N}, \Pi, K_{1}, K_{2}, \ldots, K_{N}, \varepsilon\right)$ into (17) or (16). If condition (17) or (16) is satisfied, then an MDSOF stabilizing controller and the corresponding probability rate matrix are $K_{i}=\varepsilon^{-1} \bar{K}_{i}$ and $\Pi=2 / \varepsilon \bar{\Pi}$, respectively. EXIT.

4) If $h>N_{0}$ where $N_{0}$ is the maximum number of iterations allowed, EXIT.

5) Set $h=h+1$, let $\left(X_{1}^{h}, \bar{X}_{1}^{h}, X_{2}^{h}, \bar{X}_{2}^{h}, \ldots, X_{N}^{h}, \bar{X}_{N}^{h}\right)=$ $\left(X_{1}, \bar{X}_{1}, X_{2}, \bar{X}_{2}, \ldots, X_{N}, \bar{X}_{N}\right)$, and go to Step 2).

Remark 2: Different from some other commonly used design methods involving controller reconstruction, the MDSOF gains are directly solved. An advantage of our method is that some special requirements on the desired controller can be realized. For example, we can impose some elements of the controller gain matrices to be positive, or negative, or even zeroes, which will be useful in practice.

Remark 3: All the results can be applied to mode-independent design by setting $K_{i}=K, i=1,2, \ldots, N$ in Theorem 2 and Corollary 2.
Remark 4: When some elements in the probability rate matrices of system (1) are partially known, the presented results are also applicable. In [19], a sufficient condition guaranteeing the stability was given for a class of discrete-time Markov jump linear systems with partially known transition probabilities. Therefore, the results in this technical note can be considered as an alternative development, but with a rather different spirit, of [19] for the continuous-time case.

Example 2: Consider the MDSOF design problem for system (1) with system matrices

$$
\begin{aligned}
& A_{1}=\left[\begin{array}{ll}
0 & 1 \\
0 & 1
\end{array}\right], \quad A_{2}=\left[\begin{array}{ll}
2 & 0 \\
1 & 0
\end{array}\right], \\
& B_{1}=\left[\begin{array}{l}
0 \\
1
\end{array}\right], \quad B_{2}=\left[\begin{array}{l}
1 \\
0
\end{array}\right], \\
& C_{1}=\left[\begin{array}{ll}
0 & 1
\end{array}\right], \quad C_{2}=\left[\begin{array}{ll}
1 & 0
\end{array}\right] .
\end{aligned}
$$

It is easy to test that neither $\left\{A_{1}, B_{1}, C_{1}\right\}$ nor $\left\{A_{2}, B_{2}, C_{2}\right\}$ can be stabilized by single SOF controller. Using algorithm SOFPMR and solving inequalities in (15) with constraints in (7), we have an MDSOF stabilizing controller

$$
K_{1}=[-32.1568], \quad K_{2}=[-21.1663]
$$

with the corresponding probability rate matrix

$$
\Pi=\left[\begin{array}{cc}
-5.4614 & 5.4614 \\
5.2016 & -5.2016
\end{array}\right] \text {. }
$$

Example 3: Consider the MDSOF design problem for system (1) with system matrices

$$
\begin{aligned}
& A_{1}=A_{2}=\left[\begin{array}{ccc}
-3 & 1 & 0 \\
0 & 0 & 1 \\
0 & 0 & 1
\end{array}\right], \quad B_{1}=B_{2}=\left[\begin{array}{ll}
0 & 0 \\
1 & 0 \\
0 & 1
\end{array}\right] \\
& C_{1}=\left[\begin{array}{lll}
0 & 1 & 0
\end{array}\right], \quad C_{2}=\left[\begin{array}{lll}
0 & 0 & 1
\end{array}\right] .
\end{aligned}
$$

It is also easy to test that neither $\left\{A_{1}, B_{1}, C_{1}\right\}$ nor $\left\{A_{2}, B_{2}, C_{2}\right\}$ can be stabilized by static output controller. Using algorithm SOFPMR and solving inequalities in (15) with constraints in (7), we have an MDSOF stabilizing controller

$$
K_{1}=\left[\begin{array}{c}
-7.9392 \\
1.7529
\end{array}\right], \quad K_{2}=\left[\begin{array}{c}
-7.0879 \\
-11.6218
\end{array}\right]
$$

with the corresponding probability rate matrix

$$
\Pi=\left[\begin{array}{cc}
-3.6841 & 3.6841 \\
2.0444 & -2.0444
\end{array}\right] \text {. }
$$

$$
\left[\begin{array}{cccc}
-\sum_{m=1}^{s} \alpha_{m}\left(2 X_{i}+\sum_{j=1}^{N} X_{j}\right) & \sum_{m=1}^{s} \alpha_{m} \Upsilon_{i} & \sum_{m=1}^{s} \alpha_{m}\left(I+C_{i r}^{T} \bar{K}_{i}^{T} B_{i m}^{T}\right) & \sum_{m=1}^{s} \alpha_{m}\left(I+\varepsilon A_{i m}^{T}\right) \\
* & -\sum_{m=1}^{s} \alpha_{m} \Phi & 0 & 0 \\
* & * & -\sum_{m=1}^{s} \alpha_{m} \bar{X}_{i} & 0 \\
* & * & * & -\sum_{m=1}^{s} \alpha_{m} \bar{X}_{i}
\end{array}\right]<0
$$




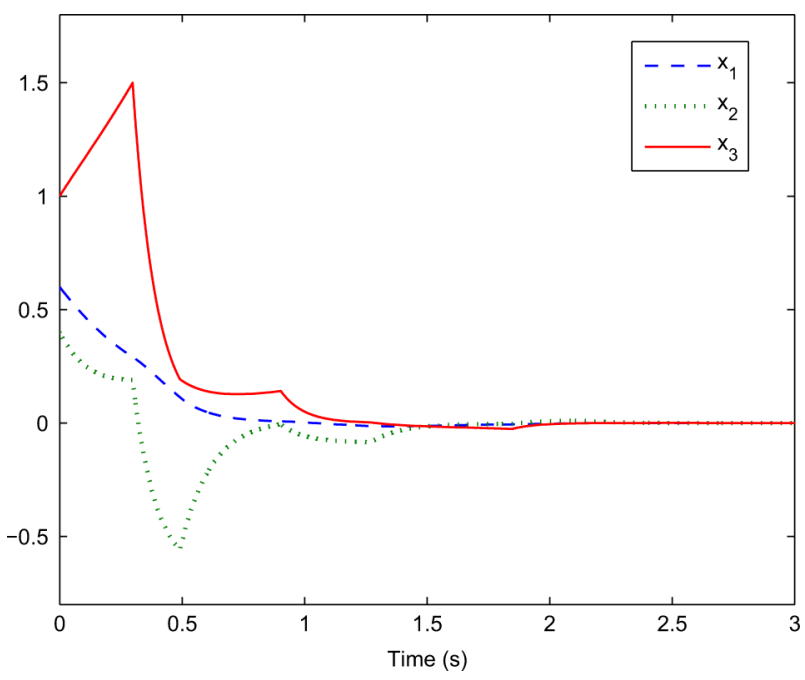

Fig. 1. Trajectory of the state variables of the closed-loop system.

Fig. 1 depicts state variable trajectories of the closed-loop system for initial value $x(0)=\left[\begin{array}{lll}0.6 & 0.4 & 1\end{array}\right]^{T}$.

\section{CONCLUSION}

In this technical note, we have studied the stabilization problem via designing probability rate matrices and SOF gains for Markovian jump linear systems. A necessary and sufficient condition with separated Lyapunov matrices and probability rates has been established. Based on this, an SOF controller and a probability rate matrix have been designed to guarantee the closed-loop stability. All the results have been extended to the polytopic uncertain case. Numerical examples have been provided to show the effectiveness of proposed approaches.

\section{REFERENCES}

[1] N. N. Krasovskii and È. A. Lidskii, "Analytical design of controllers in systems with random attributes. I. Statement of the problem, method of solving," Autom. Remote Control, vol. 22, pt. Part 1-3, pp. 1021-1294, 1961.

[2] M. Mariton, Jump Linear Systems in Automatic Control. New York: Marcel Dekker, 1990

[3] G. K. Basak, A. Bisi, and M. K. Ghosh, "Stability of a random diffusion with linear drift," J. Math. Anal. Appl., vol. 202, pp. 604-622, 1996.

[4] X. Feng, K. A. Loparo, Y. Ji, and H. J. Chizeck, "Stochastic stability properties of jump linear systems," IEEE Trans. Autom. Control, vol. 37, no. 1, pp. 38-53, Jan. 1992.

[5] Y. Ji and H. J. Chizeck, "Jump linear quadratic Gaussian control: Steady-state solution and testable conditions," Control Theory Adv. Tech., vol. 6, pp. 289-319, 1990.

[6] X. Mao, "Stability of stochastic differential equations with Markovian switching," Stoch. Process. Appl., vol. 79, pp. 45-67, 1999.

[7] J. Xiong, J. Lam, H. Gao, and D. W. C. Ho, "On robust stabilization of Markovian jump systems with uncertain switching probabilities," Automatica, vol. 41, no. 5, pp. 897-903, 2005.

[8] C. E. de Souza and M. D. Fragoso, " $\boldsymbol{H}_{\infty}$ control for linear systems with Markovian jumping parameters," Control Theory Adv. Tech., vol. 9, pp. 457-466, 1993.

[9] Y.-Y. Cao and J. Lam, "Stochastic stabilizability and $\boldsymbol{H}_{\infty}$ control for discrete-time jump linear systems with time delay," J. Franklin Inst., vol. 336, pp. 1263-1281, 1999.

[10] S. Xu and T. Chen, "Robust $\boldsymbol{H}_{\infty}$ control for uncertain discrete-time stochastic bilinear systems with Markovian switching," Int. J. Robust Nonlin. Control, vol. 15, no. 5, pp. 201-217, 2005.
[11] Y. Kang, J. F. Zhang, and S. S. Ge, "Robust output feedback $\boldsymbol{H}_{\infty}$ control of uncertain Markovian jump systems with mode-dependent time-delays," Int. J. Control, vol. 81, no. 1, pp. 43-61, 2008.

[12] S. Xu, J. Lam, and X. Mao, "Delay-dependent $\boldsymbol{H}_{\infty}$ control and filtering for uncertain Markovian jump systems with time-varying delays," IEEE Trans. Circuits Syst. I, vol. 54, no. 9, pp. 2070-2077, Sep. 2007.

[13] J. Lam, Z. Shu, S. Xu, and E. K. Boukas, "Robust $\boldsymbol{H}_{\infty}$ control of descriptor discrete-time Markovian jump systems," Int. J. Control, vol. 80, no. 3, pp. 374-385, 2007.

[14] H. Gao, J. Lam, S. Xu, and C. Wang, "Stabilization and $\boldsymbol{H}_{\infty}$ control of two-dimensional Markovian jump systems," IMA J. Math. Control Inform., vol. 21, no. 4, pp. 377-392, 2004.

[15] H. J. Chizeck, A. S. Willsky, and D. Castanon, "Discrete-time Markovian jumping linear-quadratic optimal control," Int. J.Control., vol. 43, pp. 213-231, 1986.

[16] Y. Ji and H. J. Chizeck, "Controllability, stabilizability, and continuous-time Markovian jump linear quadratic control," IEEE Trans. Autom. Control, vol. 35, no. 7, pp. 777-788, Jul. 1990

[17] Z. Mao, B. Jiang, and P. Shi, " $\boldsymbol{H}_{\infty}$ fault detection filter design for networked control systems modelled by discrete Markovian jump systems," IET Control Theory Appl., vol. 1, no. 5, pp. 1336-1343, 2007.

[18] P. Shi, E. K. Boukas, and R. K. Agarwal, "Kalman filtering for continuous-time uncertain systems with Markovian jumping parameters," IEEE Trans. Autom. Control, vol. 44, no. 8, pp. 1592-1597, Aug. 1999.

[19] L. Zhang and E. K. Boukas, "Discrete-time Markovian jump linear systems with partly unknown transition probabilities $\boldsymbol{H}_{\infty}$ filtering problem," in Proc. Amer. Control Conf., 2006, pp. 2272-2277.

[20] L. Zhang, E. K. Boukas, and J. Lam, "Analysis and synthesis of Markov jump linear systems with time-varying delays and partially known transition probabilities," IEEE Trans. Autom. Control, vol. 53, no. 10, pp. 2458-2464, Oct. 2008.

[21] D. Liberzon and A. S. Morse, "Basic problems in stability and design of switched systems," IEEE Control Syst. Mag., vol. 19, no. 5, pp. 59-70, 1999.

[22] D. D. Siljak, Decentralized Control of Complex Systems, Ser. Mathematics in Science and Engineering. Boston, MA: Academic Press Inc, 1991, vol. 184

[23] C.-C. Tsai and A. H. Haddad, "Averaging, aggregation and optima control of singularly perturbed stochastic hybrid systems," Int. J.Control., vol. 68, no. 1, pp. 31-50, 1997.

[24] L. El Ghaoui, F. Oustry, and M. AitRami, "A cone complementarity linearization algorithm for static output-feedback and related problems," IEEE Trans. Autom. Control, vol. 42, no. 8, pp. 1171-1176, Aug. 1997.

[25] O. L. V. Costa, J. B. R. Do Val, and J. C. Geromel, "Continuoustime state-feedback $\boldsymbol{H}_{2}$-control of Markovian jump linear systems via convex analysis," Automatica, vol. 35, pp. 259-268, 1999.

[26] U. Shaked, "Improved LMI representations for the analysis and the design of continuous-time systems with polytopic type uncertainty," IEEE Trans. Autom. Control, vol. 46, no. 4, pp. 652-656, Apr. 2001

[27] J. C. Geromel and P. Colaneri, "Stability and stabilization of continuous-time switched linear systems," SIAM J. Control Optim., vol. 45, no. 5, pp. 1915-1930, 2006.

[28] J. C. Geromel, P. Colaneri, and P. Bolzern, "Dynamic output feedback control of switched linear systems," IEEE Trans. Autom. Control, vol. 53, no. 3, pp. 720-733, Apr. 2008. 